\section{JTI}

JOURNAL OF

TRAUMA AND INJURY

Received: June 7, 2019

Revised: July 24, 2019

Accepted: August 6, 2019

\section{Correspondence to}

Kyung Hoon Sun, M.D., Ph.D.

Department of Emergency Medicine, Chosun University College of Medicine,

365 Pilmun-daero, Dong-gu, Gwangju

61453, Korea

Tel: $+82-62-220-3285$

Fax: +82-62-224-3501

E-mail:skhkorea@hanmail.net

\title{
The Utility of Non-Invasive Nasal Positive Pressure Ventilation for Acute Respiratory Distress Syndrome in Near Drowning Patients
}

\author{
June Hyeong Kim, M.D., Kyung Hoon Sun, M.D., Ph.D., \\ Yong Jin Park, M.D., Ph.D. \\ Department of Emergency Medicine, Chosun University College of Medicine, Gwangju, \\ Korea
}

Purpose: Near drowning refers to immediate survival after asphyxia due to submersion or immersion in water, which is a crucial public safety problem worldwide. Acute lung injury or acute respiratory distress syndrome (ARDS) is a common complication of near drowning. The purpose of this study was to investigate the feasibility and effectiveness of noninvasive nasal positive pressure ventilation (NINPPV).

Methods: This retrospective study was conducted at a tertiary emergency department. NINPPV was administered for moderate ARDS caused by submersion or immersion in patients who were older than 18 years, from January 2015 to December 2018. We collected the demographic (age, sex, length of hospital stay, and outcome), laboratory (arterial blood gas, lactate, oxygen saturation, partial pressure of oxygen divided by the fraction of inspired oxygen, complete blood count, blood urea nitrogen, and creatinine), and clinical data (acute lung injury index and ventilator failure) of the patients. A statistical analysis was performed using Statistical Package for the Social Sciences version 20.0 for Windows.

Results: NINPPV treatment was provided to 57 patients for near drowning, 45 of whom (78.9\%) were successfully treated without complications; in 12 (21.1\%), treatment was changed to invasive mechanical ventilation within 48 hours due to ARDS or acute kidney injury. NINPPV treatment was successful in 31 (75.6\%) out of 41 sea-water near drowning patients. They were more difficult to treat with NINPPV compared with the fresh-water near drowning patients $(p<0.05)$.

Conclusions: NINPPV would be useful and feasible as the initial treatment of moderate ARDS caused by near drowning.

Keywords: Near drowning; Noninvasive ventilation; Respiratory distress syndrome, adult 


\section{INTRODUCTION}

Near drowning is defined as immediate survival after asphyxia due to submersion [1]. Acute respiratory distress syndrome (ARDS) is a major complication caused by aspiration of water upon near drowning [2,3], in which permeabilities of the lung alveolar and capillary walls increase, resulting in exudates in the alveoli that cause hypoxia unresponsive to oxygen therapy due to lung injury [4]. ARDS is diagnosed when the partial pressure of oxygen divided by the fraction of inspired oxygen $\left(\mathrm{PaO}_{2} / \mathrm{FiO}_{2}\right)$ is less than 300 , and bilateral infiltrate is seen on the chest radiograph, with less than $18 \mathrm{mmHg}$ of pulmonary wedge pressure without evidence of left atrial hypertension. To treat ARDS, proper oxygenation should be ensured, with removal of the triggering factors. Mild ARDS $\left(200<\mathrm{PaO}_{2} / \mathrm{FiO}_{2} \leq 300\right)$ can be treated with non-invasive positive pressure ventilation, but high frequen$\mathrm{cy}$, low tidal volume, and high positive end expiratory pressure (PEEP) using invasive mechanical ventilation is recommended for moderate $\left(100<\mathrm{PaO}_{2} / \mathrm{FiO}_{2} \leq 200\right)$ and severe $\left(\mathrm{PaO}_{2} / \mathrm{FiO}_{2} \leq 100\right)$ ARDS [5]. Invasive mechanical ventilation and face mask or helmet type non-invasive ventilation has been used to treat ARDS, but the effect of non-invasive nasal positive pressure ventilation (NINPPV) using high-flow nasal cannula has not been reported as a treatment modality for moderate ARDS [6,7]. We performed this study to investigate the effect of NINPPV to treat moderate ARDS caused by near drowning.

\section{METHODS}

For this retrospective study, data of patients who visited the emergency room for near drowning in Gwangju, South Korea between January 2015 and December 2018 were collected. This study was approved by the Institutional Review Board (IRB No. CHOSUN 2019-05-010-001). The included patients were 18 years of age or older who had a consciousness level of 12 or above on the Glasgow coma scale (GCS) and visited Chosun University Hospital immediately, without admission to any other hospital after near drowning. The exclusion criteria were age below 18 years, GCS score below 12. A total of 68 patients were admitted to Chosun University Hospital during the study period. We enrolled 57 patients and excluded 11 based on the exclusion criteria. The patients were classified based on near drowning in the sea or fresh water (river, reservoir, bath, etc.) (Fig. 1). NINPPV was administered to all patients with moderate ARDS of 12 or above

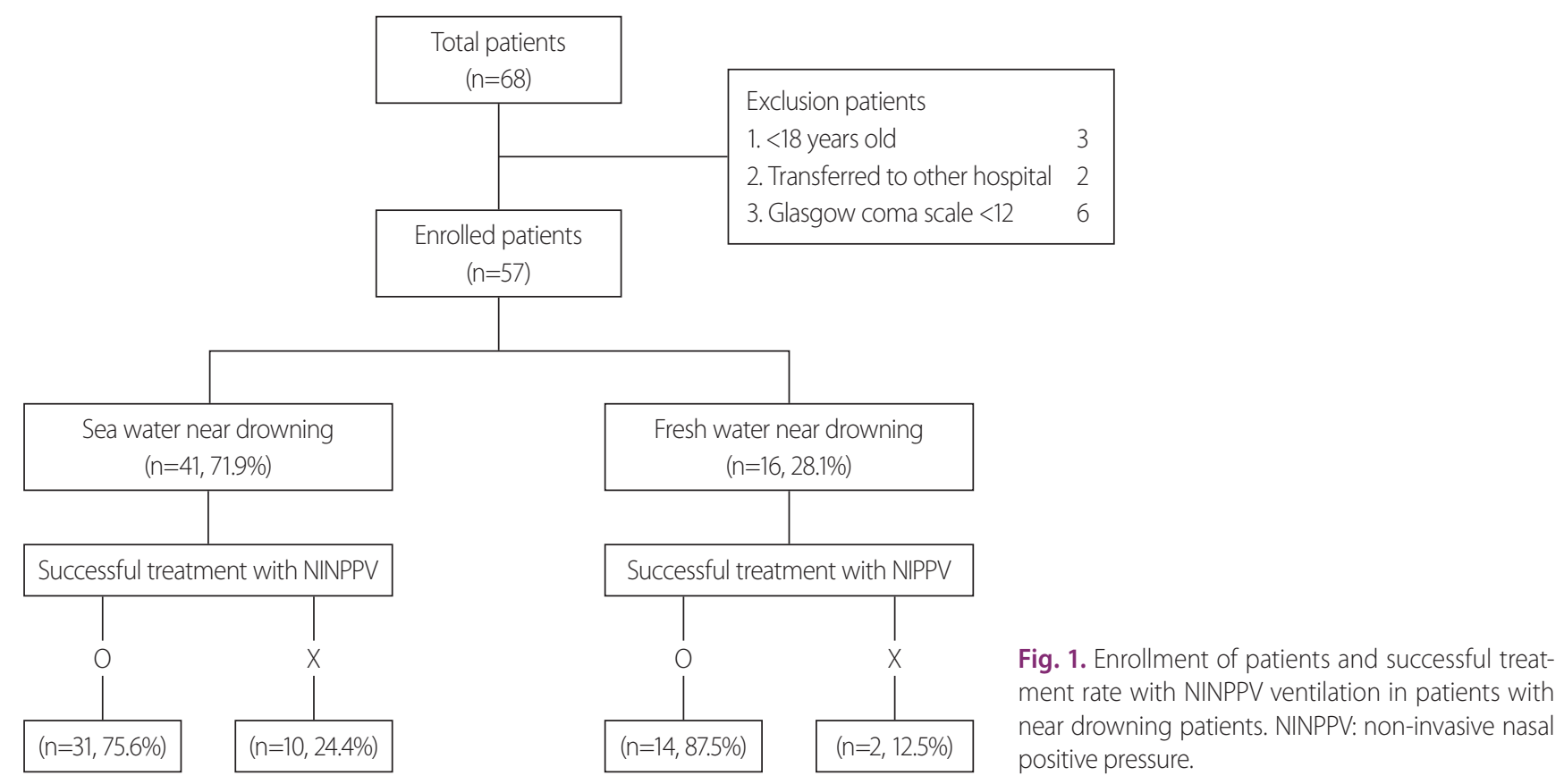


GCS score (initial setting: $\mathrm{FiO}_{2}$, 1.0; oxygen flow, $50 \mathrm{~L} / \mathrm{min}$; PEEP, 4-5 $\mathrm{cmH}_{2} \mathrm{O}$ ). The patients were treated with piperacillin-tazobactam based on creatinine clearance. We investigated the number of patients, sex, age, GCS score, Simplified Acute Physiology Score (SAPS) II, acute physiology and chronic health evaluation (APACHE) II score, Modified Early Warning Score (MEWS), length of hospital stay and ventilator outcome (Table 1). Arterial blood gas, lactate, complete blood count, blood urea nitrogen, creatinine, and creatinine kinase were investigated for 48 hours to determine the effectiveness of NINPPV treatment (Table 2). The severity of patient status was determined by SAPS II, APACHE II score, and MEWS. The failure of NINPPV treatment was defined as the use of an invasive mechanical ventilator or extracorporeal

Table 1. Characteristics of near drowning patients and outcomes

\begin{tabular}{|lccc|}
\hline & Sea water & Fresh water & $\boldsymbol{p}$-value \\
\hline Patients (male) & $41(35)$ & $16(13)$ & 0.128 \\
Age (years) & $27.5 \pm 4.8$ & $29.1 \pm 6.2$ & 0.646 \\
GCS & $13.1 \pm 1.2$ & $13.5 \pm 0.8$ & 0.544 \\
SAPS II & $42.8 \pm 3.8$ & $43.5 \pm 2.5$ & 0.557 \\
APACHE II & $13.6 \pm 4.1$ & $14.2 \pm 2.6$ & 0.356 \\
MEWS & $4.5 \pm 2.2$ & $4.8 \pm 1.8$ & 0.385 \\
Length of HD (day) & $13.8 \pm 6.4$ & $10.5 \pm 4.9$ & 0.045 \\
Ventilator outcomes & & & \\
Successful treatment & & $45(78.9)$ & \\
$\quad$ with NINPPV & & & \\
NINPPV & $31(75.6)$ & $14(87.5)$ & 0.048 \\
Invasive mechanical & $10(24.4)$ & $2(12.5)$ & 0.047 \\
$\quad$ ventilator & & & \\
ECMO & $2(4.8)$ & $0(0)$ & 0.530 \\
CRRT & $2(4.8)$ & $0(0)$ & 0.530 \\
Patients outcome & & & NS \\
Discharge & $39(95.1)$ & $16(100.0)$ & NS \\
Death/hopeless discharge & $2(4.9)$ & $0.0)$ & $\mathrm{NS}$ \\
\hline
\end{tabular}

Values are presented as number (\%) or mean \pm standard deviation. GCS: Glasgow coma scale, SAPS: simplified acute physiology score, APACHE: acute physiology chronic health evaluation, MEWS: modified early warning score, HD: hospital day, NINPPV: non-invasive nasal positive pressure ventilation, ECMO: extracorporeal membrane oxygenation, CRRT: continuous renal replacement therapy, NS: not significant. membrane oxygenator (ECMO), and clinical improvement was associated with the length of hospital stay. A statistical analysis was performed using Statistical Package for the Social Sciences version 20.0 (SPSS, IBM Corp., Armonk, NY, USA). Continuous variables were expressed as mean \pm standard deviation, and the frequency and percentage of each item were the categorical variables. To verify the statistical significance, chi-squared or Fisher's exact test was used for categorical variables and Student's $t$-test or Mann-Whitney $U$ test for continuous variables. Linear regression and repeated measures analyses were used for the continuous time variables of $\mathrm{PaO}_{2} / \mathrm{FiO}_{2}$ and peripheral capillary oxygen saturation $\left(\mathrm{SpO}_{2}\right)$. A $p$-value $<0.05$ was considered statistically significant.

\section{RESULTS}

NINPPV treatment was successful in 45 out of 57 patients (78.9\%). The initial NINPPV was changed to invasive mechanical ventilation within 48 hours in 12 patients (21.1\%) because of aggravated ARDS or acute kidney injury (AKI) (Table 1, Fig. 1). Two patients in the sea-water near drowning (SWND) group required continuous renal replacement therapy (CRRT) because of AKI, and two patients required ECMO because of failure of invasive mechanical ventilation.

We compared the effect of NINPPV treatment between SWND and fresh-water near drowning (FWND) by dividing the patients into SWND and FWND groups. The characteristics of patients who visited the emergency room for near drowning were analyzed, which revealed no significant differences in age or sex of the patients between the SWND and FWND groups. Patients' severity status, as determined by SAPS II, APACHE II score, and MEWS, did not significantly differ between the two groups (Table 1). NINPPV treatment was successful in 31 out of 41 patients $(75.6 \%)$ in the SWND group and in 14 out of 16 patients $(87.5 \%)$ in the FWND group $(p=0.048)$. To compare the treatment effectiveness between the two groups, blood tests were analyzed for 48 hours. The test results showed significantly improved $\mathrm{pH}, \mathrm{PaO}_{2}$, lactate, white blood cell and platelet counts, and creatinine kinase and creatinine levels in the FWND 
Table 2. Laboratory features in near drowning patients between sea water and fresh water

\begin{tabular}{|c|c|c|c|c|c|c|c|}
\hline & \multicolumn{2}{|c|}{ Initial (on arrival) } & \multicolumn{2}{|c|}{24 hours } & \multicolumn{2}{|c|}{48 hours } & \multirow{2}{*}{$p$-value } \\
\hline & Sea water & Fresh water & Sea water & Fresh water & Sea water & Fresh water & \\
\hline \multicolumn{8}{|l|}{ Arterial blood gas } \\
\hline $\mathrm{pH}$ & $7.1(6.9-7.2)$ & $7.2(6.8-7.3)$ & $7.3(7.0-7.4)$ & $7.3(7.1-7.4)$ & $7.3(6.9-7.3)$ & $7.4(7.2-7.5)$ & 0.04 \\
\hline $\mathrm{pO}_{2}(\mathrm{mmHg})$ & $82(78-88)$ & $85(72-90)$ & $86(84-95)$ & $90(82-94)$ & $91(89-96)$ & $90(88-98)$ & 0.02 \\
\hline $\mathrm{pCO}_{2}(\mathrm{mmHg})$ & $52(48-58)$ & $51(48-55)$ & $48(42-55)$ & $46(41-50)$ & $46(42-54)$ & $43(40-48)$ & 0.25 \\
\hline $\mathrm{HCO}_{3}(\mathrm{mmol} / \mathrm{L})$ & $16(14-18)$ & $18(14-20)$ & $22(18-28)$ & $20(16-22)$ & $20(16-22)$ & $24(20-26)$ & 0.15 \\
\hline Lactate (mmol/L) & $3.8(2.5-4.5)$ & $2.4(2.2-3.6)$ & $5.8(3.5-7.5)$ & $3.9(2.8-4.8)$ & $4.6(3.2-5.9)$ & $4.4(2.5-4.6)$ & 0.01 \\
\hline \multicolumn{8}{|l|}{ Complete blood counts } \\
\hline Hemoglobin (g/dL) & $14(13-15)$ & $14(12-15)$ & $14(13-16)$ & $13(12-15)$ & $13(12-15)$ & $14(12-15)$ & NS \\
\hline Platelets $(\times 1,000 / \mu \mathrm{L})$ & $251(224-262)$ & $244(218-252)$ & $211(185-225)$ & $208(188-223)$ & $198(182-214)$ & $202(192-226)$ & 0.01 \\
\hline White blood cell $(\times 1,000 / \mu \mathrm{L})$ & $12.8(11.6-14.2)$ & $13.8(12.1-15.2)$ & $18.8(15.6-20.8)$ & $16.4(14.2-18.2)$ & $16.2(14.6-18.6)$ & $14.2(13.6-16.7)$ & 0.02 \\
\hline \multicolumn{8}{|l|}{ Chemistry } \\
\hline Sodium (mEq/L) & 142 (139-144) & $138(135-142)$ & $146(141-148)$ & $137(135-142)$ & $145(139-149)$ & $140(135-144)$ & NS \\
\hline Potassium (mEq/L) & $3.7(3.5-3.8)$ & $3.6(3.5-3.9)$ & $4.4(3.9-4.8)$ & $3.6(3.3-3.7)$ & $4.7(4.2-5.0)$ & $3.9(3.6-4.5)$ & 0.15 \\
\hline Chloride (mEq/L) & $108(105-110)$ & 100 (98-104) & $100(107-112)$ & 99 (95-104) & 104 (99-108) & $98(95-104)$ & NS \\
\hline Creatinine kinase $(\mathrm{U} / \mathrm{L})$ & $200(125-263)$ & $189(125-205)$ & $668(525-863)$ & $560(433-742)$ & 786 (725-930) & $586(325-692)$ & 0.03 \\
\hline Blood urea nitrogen $(\mathrm{mg} / \mathrm{dL})$ & $18(14-20)$ & $14(12-16)$ & $26(22-34)$ & $23(19-26)$ & $28(25-32)$ & $26(22-29)$ & 0.25 \\
\hline Creatinine (mg/dL) & $0.8(0.7-1.0)$ & $0.9(0.8-1.2)$ & $1.1(0.7-1.4)$ & $1.0(0.8-1.3)$ & $1.3(0.9-1.7)$ & $1.2(0.9-1.5)$ & 0.01 \\
\hline
\end{tabular}

Values are presented as median (interquartile range).

NS: not significant.

group (Table 2). We observed both groups for 48 hours for changes in $\mathrm{PaO}_{2} / \mathrm{FiO}_{2}$ and $\mathrm{SaO}_{2}$ over time, which showed significant improvement in FWND group compared to SWND group after 48 hours (Table 3, Fig. 2). The SWND group patients were more difficult to treat with NINPPV compared with the FWND group $(p<0.05)$.

\section{DISCUSSION}

This is the first study on the effect of NINPPV treatment on moderate ARDS caused by near drowning. Near drowning is defined as immediate survival after asphyxia due to submersion and may cause various complications, such as ARDS, pneumonia, sepsis, and AKI [1,3,8]; among these, ARDS due to increased permeabilities of lung alveolar-capillary membranes is common and classified as severe $\left(\mathrm{PaO}_{2} / \mathrm{FiO}_{2} \leq 100\right)$, moderate $\left(100<\mathrm{PaO}_{2} / \mathrm{FiO}_{2} \leq 200\right)$, and mild $\left(200<\mathrm{PaO}_{2} / \mathrm{FiO}_{2} \leq 300\right)$ [4]. Adequate oxygen- ation is the most important aspect in ARDS management. Therefore, ARDS patients often require non-invasive positive pressure or invasive mechanical ventilation. In general, low tidal volume, high frequency, and high PEEP with invasive mechanical ventilation are recommended for moderate and severe ARDS [5,9]. NIPPV has been administered to treat ARDS, but this approach is controversial, as its prognosis has been suggested to be poor [7]. In a recent study, the success rate of NIPPV treatment was high for mild ARDS but low for moderate and severe ARDS [6]. The purpose of this study was to evaluate the therapeutic effect of NINPPV in moderate ARDS patients who could receive NIPPV based on their consciousness levels. We administered NINPPV instead of using a helmet or face mask as the method of NIPPV. To administer NINPPV, a high-flow nasal cannula was used, through which high $\mathrm{FiO}_{2}$ and PEEP can be supplied with minimal patient discomfort [10]. In addition, NINPPV reduces the rate of endotracheal intubation, which can lead to com- 
Table 3. Time flows of $\mathrm{PaO}_{2} / \mathrm{FiO}_{2}$ and $\mathrm{SpO}_{2}$ values

\begin{tabular}{|llll|}
\hline & Sea water & Fresh water & $p$-value \\
\hline $\mathrm{PaO}_{2} / \mathrm{FiO}_{2}$ & & & \\
Initial & $122(118-134)$ & $138(122-142)$ & 0.763 \\
2 hours & $118(106-132)$ & $130(120-144)$ & 0.832 \\
12 hours & $106(102-122)$ & $138(120-142)$ & 0.589 \\
24 hours & $130(126-158)$ & $154(136-158)$ & 0.214 \\
48 hours & $152(150-165)$ & $162(150-178)$ & 0.014 \\
$\mathrm{SaO}_{2}(\%)$ & & & \\
Initial & $88(80-92)$ & $90(85-95)$ & 0.426 \\
2 hours & $90(85-93)$ & $91(85-94)$ & 0.751 \\
12 hours & $89(84-94)$ & $92(84-97)$ & 0.665 \\
24 hours & $90(86-93)$ & $94(88-97)$ & 0.229 \\
48 hours & $93(89-95)$ & $95(93-98)$ & 0.032 \\
\hline
\end{tabular}

Values are presented as median (interquartile range).

plications, such as pneumonia, tracheal stenosis, and ventilator-induced lung injury [11]. We initiated NINPPV treatment, initially set to an $\mathrm{FiO}_{2}$ of 1.0 , oxygen flow of $50 \mathrm{~L} / \mathrm{min}$, and PEEP of 4-5 $\mathrm{cmH}_{2} \mathrm{O}$, and investigated the progression of $\mathrm{PaO}_{2} / \mathrm{FiO}_{2}$ and $\mathrm{SaO}_{2}$ (Table 2, Fig. 2). The success rate of treatment was greater than $75 \%$, which was better than the results of a previous study [6]. This was attributed to the early administration of high oxygen concentration and PEEP to prevent complications due to hypoxia. In a previous study, comorbidities, one of the causes of NIPPV failure in ARDS patients, increased with age. There are many young individuals and travelers because there are many coasts and hot springs around the hospital as the local landmarks. Therefore, it could be considered that because the mean age of the patients was low (twenties) compared to that in the previous study (sixties), the prognosis may have been favorable. Moreover, in the previous study, all patients with acute hypoxemic respiratory failure were included. However, in this study, patients with ARDS only due to near drowning were included. Another study showed that initially, it was difficult to distinguish near drowning-related lung abnormalities from bacterial pneumonia or chemical pneumonitis, which did not affect the mortality rate even if the result was bacterial pneumonia [12]. Therefore, the results of this study might be better than the results of previous successful treatment

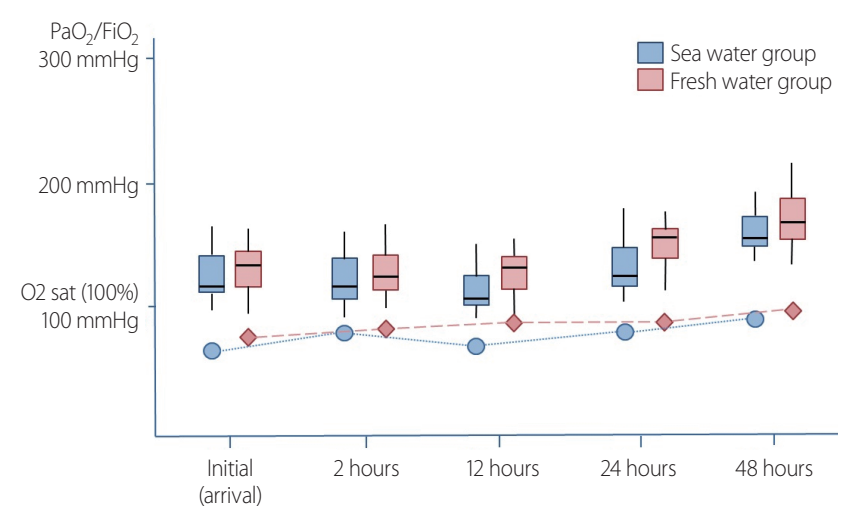

Fig. 2. Time flow of changes in $\mathrm{PaO}_{2} / \mathrm{FiO}_{2}$ and $\mathrm{SaO}_{2}$ over time for 48 hours in sea water near drowning group and fresh water near drowning group.

\section{of ARDS with NIPPV.}

In this study, near drowning patients were classified into SWND and FWND groups, and the clinical differences were investigated. A previous study showed that seawater has high osmotic pressure, thus causing the movement of a greater volume of water from the blood to the alveoli upon aspiration, which can lead to hypovolemia, hypernatremia, hyperchloremia, and damage to the alveolar and capillary membranes, resulting in pulmonary edema of higher severity. In contrast, freshwater can cause hypervolemia and hyponatremia because of low osmotic pressure $[2,13]$. This study showed no significant differences in severity between the two groups at the time of admission in the emergency room, but the treatment period was significantly longer in the SWND group, and the percentage of patients who switched from NINPPV to invasive mechanical ventilation was significantly higher. The blood test results 48 hours after admission showed significant differences in the rate of improvement between the two groups. These results suggest that the treatment for SWND group patients is more difficult and the prognosis is worse, similar to the results of previous studies. However, unlike in previous studies, levels of blood electrolytes, such as sodium and chloride, did not differ significantly between the two groups. These results may be due to the inclusion of moderate ARDS patients who did not aspirate large amounts of salt water. In two patients with SWND, NINPPV and conventional invasive mechanical ventilation treatment failed, so ECMO treatment 
was performed. The findings of patients' chest radiographs deteriorated, and despite optimal ventilatory care, such as $\mathrm{FiO}_{2}>90 \%$ and high $\mathrm{PEEP}, \mathrm{PaO}_{2} / \mathrm{FiO}_{2}<100$ was maintained for over 6 hours and veno-venous ECMO was administered, which is used to provide lung rest by permitting reduced ventilator settings, decreased concentrations of inspired oxygen, and removal of carbon dioxide. Therefore, ECMO may be a successful treatment modality in near drowning patients with pulmonary failure refractory to conventional invasive ventilation therapy [14]. In some studies, the results of drowning-induced ARDS were demonstrated to be successful with ECMO, and all the patients were weaned and discharged successfully $[15,16]$. Two patients who were treated with ECMO were successfully weaned, but one patient was discharged to another hospital for personal reasons without adequate recovery, who then developed worsening of pneumonia and died. Because moderate ARDS patients were investigated in this study, only a few patients received ECMO, but worsening of the clinical course becomes an indication for ECMO, and aggressive treatment may be helpful. Two patients underwent CRRT because their creatinine levels increased by more than two-fold during the hospital stay, and the urine output was less than $0.3 \mathrm{~mL} / \mathrm{kg} / \mathrm{h}$ despite adequate fluid therapy. AKI in near drowning patients is caused by hypoxic renal injury, reduced renal blood flow, and systemic inflammatory response [17-19]. However, since patients who require CRRT are uncommon, AKI must be mild and is probably caused by acute tubular necrosis [8,17-19]. Unlike previous studies, we did not perform renal biopsy to differentiate the causes of AKI. However, because the renal function recovered within 4 and 6 days of CRRT, respectively, renal damage might not have been serious.

One limitation of this study was the small sample size because of the single-institutional design; the clinical course in the FWND group was not analyzed based on the contamination degree of the aspirated water. We could not present the strains of bacteria in patients with pneumonia because early bacterial cultures were not performed in many cases, and infections were often suspected after admission (hospital-acquired infection). Further studies of the prognostic significance according to the bacterial strains of pneumonia are needed. Second, one
SWND patient undergoing invasive mechanical ventilator treatment was hopelessly discharged to another hospital at the request of their caregiver. Six months later, we called the family and discovered that the patient had died, but we could not determine the exact cause of death. Third, we did not investigate the therapeutic effect of NINPPV in patients with mild or severe ARDS because the study subjects only had moderate ARDS.

\section{CONCLUSION}

Invasive mechanical ventilation is generally recommended, and the NIPPV approach is controversial for moderate ARDS caused by near drowning. However, our findings suggest that NIPPV using NINPPV can be administered as first-line treatment because it was shown to be effective in the patients in this study.

\section{ACKNOWLEDGEMENTS}

This study was supported by research fund from Chosun University Hospital, 2018.

\section{REFERENCES}

1. Moon RE, Long RJ. Drowning and near-drowning. Emerg Med (Fremantle) 2002;14:377-86.

2. Azzorina R, Camera GL, Messina A, Fioino LS, Chiarenza F, Francesco AD, et al. Pathophysiology, prevention and treatment of drowing syndrome. Acta Medica Mediterranea 2011;27:31-3.

3. Szpilman D, Bierens JJ, Handley AJ, Orlowski JP. Drowning. N Engl J Med 2012;366:2102-10.

4. Kim JH. New definition of acute respiratory distress syndrome. Korean J Crit Care Med 2013;28:10-6.

5. Weiss CH, McSparron JI, Chatterjee RS, Herman D, Fan E, Wilson $\mathrm{KC}$, et al. Summary for clinicians: mechanical ventilation in adult patients with acute respiratory distress syndrome clinical practice guideline. Ann Am Thorac Soc 2017;14:1235-8.

6. Bellani G, Laffey JG, Pham T, Madotto F, Fan E, Brochard L, et al. Noninvasive ventilation of patients with acute respiratory distress syndrome. Insights from the LUNG SAFE study. Am J 
Respir Crit Care Med 2017;195:67-77.

7. Mosier JM, Hypes C, Joshi R, Whitmore S, Parthasarathy S, Cairns CB. Ventilator strategies and rescue therapies for management of acute respiratory failure in the emergency department. Ann Emerg Med 2015;66:529-41.

8. Gorelik Y, Darawshi S, Yaseen H, Abassi Z, Heyman SN, Khamaisi M. Acute renal failure following near-drowning. Kidney Int Rep 2018;3:833-40.

9. Fan E, Del Sorbo L, Goligher EC, Hodgson CL, Munshi L, Walkey AJ, et al. An official American Thoracic Society/European Society of Intensive Care Medicine/Society of Critical Care Medicine clinical practice guideline: mechanical ventilation in adult patients with acute respiratory distress syndrome. Am J Respir Crit Care Med 2017;195:1253-63.

10. Parke RL, Eccleston ML, McGuinness SP. The effects of flow on airway pressure during nasal high-flow oxygen therapy. Respir Care 2011;56:1151-5.

11. Ou X, Hua Y, Liu J, Gong C, Zhao W. Effect of high-flow nasal cannula oxygen therapy in adults with acute hypoxemic respiratory failure: a meta-analysis of randomized controlled trials. CMAJ 2017;189:E260-7.

12. Cerland L, Mégarbane B, Kallel H, Brouste Y, Mehdaoui H, Resiere D. Incidence and consequences of near-drowningrelated pneumonia-A descriptive series from Martinique, French
West Indies. Int J Environ Res Public Health 2017;14:E1402.

13. Jin F, Li C. Seawater-drowning-induced acute lung injury: from molecular mechanisms to potential treatments. EXP Ther Med 2017;13:2591-8.

14. Kim KI, Lee WY, Kim HS, Jeong JH, Ko HH. Extracorporeal membrane oxygenation in near-drowning patients with cardiac or pulmonary failure. Scand J Trauma Resusc Emerg Med 2014;22:77.

15. Stachon P, Kalbhenn J, Walterspacher S, Bode C, Staudacher D. Near-drowning with good outcome after ECMO-therapy and therapeutic hypothermia despite 20 minutes of anoxia and 16 hours of hypoxia. Dtsch Med Wochenschr 2017;142:596-600.

16. Weuster M, Haneya A, Panholzer B, Klüter T, van der Brelie M, van Laak $U$, et al. The use of extracorporeal membrane oxygenation systems in severe accidental hypothermia after drowning: a centre experience. ASAIO J 2016;62:157-62.

17. Amir A, Lee YL. A case of acute kidney injury by near-drowning. Malays Fam Physician 2013;8:34-6.

18. Seong EY, Rhee H, Lee N, Lee SJ, Song SH, Lee DW, et al. A case of severe acute kidney injury by near-drowning. J Korean Med Sci 2012;27:218-20.

19. Alp A, Akdam H, Meteoğlu I, Ünsal A, Akar H, Yeniçerioğlu Y. Acute kidney injury after near drowning: the way from the beach to hemodialysis. Hemodial Int 2016;20:E1-4. 